\title{
Influence of Social Isolation and Loneliness on the Prognosis of Advanced Lung Cancer Patients: A Prospective Cohort Study
}

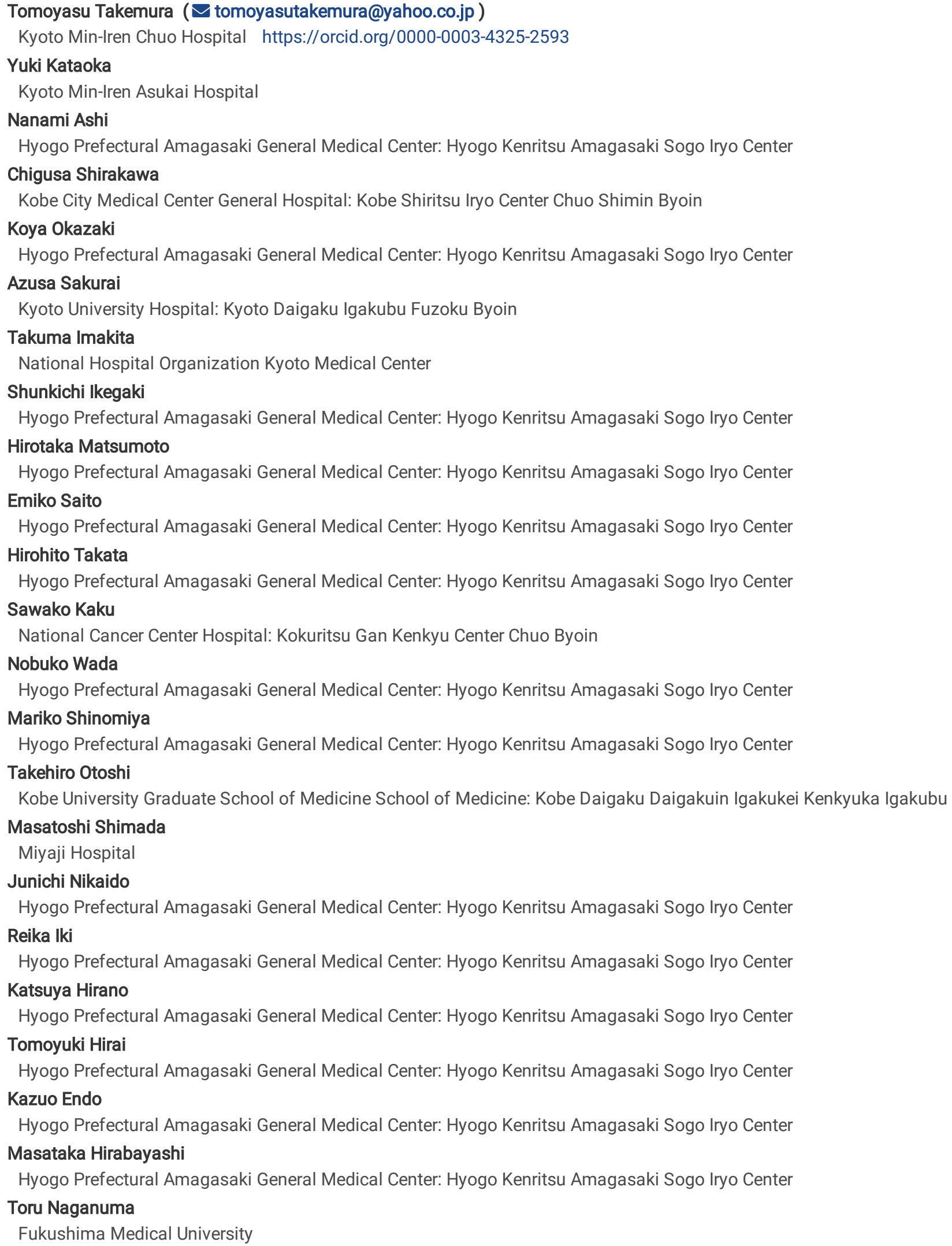


Research Article

Keywords: social determinants of health (SDHs), social isolation, loneliness, advanced lung cancer, overall survival, prospective cohort study

Posted Date: February 8th, 2022

DOI: https://doi.org/10.21203/rs.3.rs-1220364/v1

License: @ (i) This work is licensed under a Creative Commons Attribution 4.0 International License. Read Full License

Version of Record: A version of this preprint was published at Supportive Care in Cancer on April 13th, 2022. See the published version at https://doi.org/10.1007/s00520-022-07026-1. 


\section{Abstract \\ Purpose}

The purpose of this study was to investigate the impact of social isolation and loneliness on the overall survival and death at home in patients with lung cancer.

\section{Methods}

This prospective cohort study was conducted in a Japanese tertiary hospital. The enrollment period was from April 2018 to March 2020. Patients with pathologically diagnosed advanced lung cancer were included in this study. The primary outcome was overall survival, whereas the secondary outcome was death at home. The exposures were social isolation and loneliness.

\section{Results}

A total of 211 patients were enrolled and divided into two groups and further into quartiles according to their social isolation and loneliness level, respectively. The hazard ratios of social isolation were 1.65 ( $95 \%$ confidence interval; 1.12 to 2.44 ) and 1.87 (95\% confidence interval; 1.15 to 3.03$)$ in the univariate analysis, while 1.40 (95\% confidence interval; 0.92 to 2.13 ) in the multivariate analysis with complete case and multiple imputation. The odds ratio of death at home with social isolation was 3.47 ( $95 \%$ confidence interval; 1.08 to 11.1 ) in the multivariate analysis with multiple imputation. Loneliness was not associated with overall survival or death at home.

\section{Conclusions}

Our study suggests that social isolation may be related to overall survival and death at home among patients with advanced lung cancer. More attention should be given to such patients at the time of diagnosis.

\section{Introduction}

Non-medical factors, such as social factors, that influence people's health are called the social determinants of health (SDHs) [1]. In 2011, during an international conference held in Rio de Janeiro, the World Health Organization (WHO) highlighted SDHs as factors that should be addressed to promote health equity [2]. The main focus of SDH research has been on social interactions, including social isolation and Ioneliness which can affect a person's living environment, service access, and physical activity [3]. Macro-level factors such as race, ethnicity, and socioeconomic status are upstream SDH factors, and social isolation and loneliness are also considered to be in this category [3]. Health behaviors, education, and economic status are midstream factors [3] and are influenced by the upstream factors. Prevention, diagnosis, and treatment are downstream factors that are directly related to health. The upstream factors influence the midstream factors, and midstream factors influence the downstream factors [3]. Therefore, all the factors should be considered separately. For example, a cross-sectional study showed that the population attributable risk for cancer screening had a $38 \%$ difference between the lowest and highest advantaged socioeconomic groups [4]. A case-control study showed that Black and Hispanic patients had a higher attributable risk for inadequately treated hypertension in intracerebral hemorrhage compared to Caucasian patients [5].

Social isolation is defined as "the objectively quantified shortfall in an individual's social relationships often measured in terms of social network size and diversity or frequency of contacts." Loneliness is defined as "a perceived deficit between the actual and desired quality or quantity of relationships." [6]. A systematic review showed that social isolation and loneliness were associated with higher all-cause mortality rates, depressive symptoms, and poorer quality of life in the general population $[7,8]$. Previous cohort studies have shown that living in highpoverty areas, having no insurance, and having a low level of education were prognostic factors among lung cancer patients [9-12]. In contrast, few studies have assessed social isolation and loneliness in patients with cancer [3]. Our cross-sectional study investigated the relationship between social isolation and loneliness with diagnosis and treatment choice in patients with lung cancer [13]. We hypothesized that social isolation and loneliness potentially influenced the prognosis of patients with advanced lung cancer. The purpose of this study was to investigate the relationship between social isolation and loneliness with the overall survival (OS) and death at home in Japanese patients with advanced lung cancer.

\section{Materials And Methods Study design and setting}


This prospective cohort study was in accordance with the Strengthening the Reporting of Observational Studies in Epidemiology statement. This study was conducted at a tertiary referral hospital in Japan (Hyogo Prefectural Amagasaki General Medical Center). The study protocol and other results have been published previously $[14,15]$. The study was conducted in accordance with the Declaration of Helsinki. All patients provided written informed consent before participation.

\section{Participants and variables}

The eligibility criteria were as follows: (i) patients who were pathologically diagnosed for lung cancer from April 2018 to March 2020, (ii) stage IIIA or higher based on the 8th edition of the American Joint Commission on Cancer and Union for Cancer Control tumor node metastasis (TNM) classification stage [16], (iii) patients who were considered unsuitable for radical surgery (to avoid omission of data with surgery), (iv) patients who had not been treated for lung cancer in the past two months (since there were some cases in which urgent treatment was started before the patient was judged for inclusion, we included the patients within two months after the start of treatment), and ( $v$ ) written informed consent was obtained from the patient for participation. The exclusion criteria were as follows: (i) inability to complete the questionnaire due to dementia or psychiatric illness, and (ii) those considered unsuitable for the study by the physician.

Exposures were defined as social isolation and loneliness. Social isolation was assessed using the Japanese version of the Lubben Social Network Scale (LSNS-6) [17]. Since the Japanese version did not have a threshold, we used the cut-off value of the English version (12 points out of 30) [17]. Loneliness was assessed using the third Japanese version of the University of California, Los Angeles (UCLA) Loneliness Scale (3-12 points) [18]. The UCLA scale was revised and converted into three questions to facilitate patient responses [19]. Since the scale did not have a clear cutoff point, participants were categorized into quartiles (low to high loneliness) to create a relatively homogeneous group.

The primary and secondary outcomes were OS and death at home, respectively. Potential confounders included sex, age, smoking status, and insurance status (welfare or other). Education status, employment status, dementia, Eastern Cooperative Oncology Group performance status, histology, TNM classification stage [16], targetable driver gene mutation, programmed death-ligand 1 (PD-L1) tumor proportion score, and initial treatment. Dementia was assessed using the Life Function Evaluation for Care Provision [20].

\section{Statistical analysis}

The protocol for this study required the enrollment of 300 patients. However, due to inadequate enrollment, recruitment was withdrawn two years later. To reduce information bias, the clinical research coordinator conducted the data collection.

The Kaplan-Meier method was used to estimate OS, and the differences were evaluated using the log-rank test. Cox proportional-hazards models were used for the univariate and multivariate analyses of the prognostic factors. Odds ratios and confidence intervals with death at home were calculated using a logistic regression model. We conducted a complete case analysis and multiple imputation to analyze the missing data for exposure and outcome variables and combined 20 imputed datasets using Rubin's rule [21]. Statistical significance was set at $\mathrm{P}<0.05$.

\section{Results}

\section{Overall survival}

A total of 211 patients with advanced lung cancer were analyzed (Figure 1). We observed the patients until March 2021. Patient characteristics are summarized in Table 1 . The social isolation absent group included 135 patients $(64 \%)$, and the present group included $73(35 \%)$ patients. A total of six patients, three from each group, were excluded due to incomplete questionnaires. Patients were categorized into quartiles 1 ( $\leq 3$ points), 2 (4-5 points), 3 (6-7 points), and 4 (8-12 points) based on the UCLA loneliness scale score. Each group included 56 (27\%) patients, 33 (16\%) patients, $81(38 \%)$ patients, and $37(18 \%)$ patients, respectively. Of these, four patients were not included in each group due to incomplete questionnaires. Except for the driver gene mutations and PD-L1 that were not required for all patients, the percentage of missing data for each confounding factor was less than $3 \%$, due to incomplete questionnaires. 
Table 1

Characteristics of patients

\begin{tabular}{|c|c|c|c|c|c|c|c|}
\hline \multirow[b]{2}{*}{ Characteristic } & \multirow[b]{2}{*}{$\begin{array}{l}\text { Overall, } \mathrm{N}= \\
211\end{array}$} & \multicolumn{2}{|c|}{ Social Isolation* } & \multicolumn{2}{|l|}{ Loneliness $^{\dagger}$} & \multirow[b]{2}{*}{$\begin{array}{l}\text { q3 }(>5, \leq 7), N \\
=81\end{array}$} & \multirow[b]{2}{*}{$\begin{array}{l}\mathrm{q} 4(\geq 8), \mathrm{N}= \\
37\end{array}$} \\
\hline & & $\begin{array}{l}\text { Absent, } N= \\
135\end{array}$ & $\begin{array}{l}\text { Present, N } \\
=73\end{array}$ & $\begin{array}{l}q 1(\leq 3), N \\
=56\end{array}$ & $\begin{array}{l}\mathrm{q} 2(>3, \leq 5), \mathrm{N} \\
=33\end{array}$ & & \\
\hline Age (years) & $71(67,77)$ & $72(68,77)$ & $70(65,79)$ & $72(68,81)$ & $73(68,79)$ & $70(66,76)$ & $71(64,77)$ \\
\hline Gender (male) & $166(79 \%)$ & $103(76 \%)$ & $61(84 \%)$ & 37 (66\%) & $28(85 \%)$ & $63(78 \%)$ & $34(92 \%)$ \\
\hline \multicolumn{8}{|l|}{$\begin{array}{l}\text { (Current smoker or Ex- } \\
\text { smoker) }\end{array}$} \\
\hline (Missing) & 33 & 27 & 6 & 12 & 4 & 11 & 5 \\
\hline Performance status $(\geq 2)$ & $51(24 \%)$ & $28(21 \%)$ & $21(29 \%)$ & $10(18 \%)$ & $10(30 \%)$ & $20(25 \%)$ & $11(30 \%)$ \\
\hline \multicolumn{8}{|l|}{$\begin{array}{l}\text { (University or higher-level } \\
\text { degree) }\end{array}$} \\
\hline (Missing) & 1 & 1 & 0 & 1 & 0 & 0 & 0 \\
\hline Insurance (Welfare) & $29(14 \%)$ & $11(8.1 \%)$ & $18(25 \%)$ & $4(7.1 \%)$ & $3(9.1 \%)$ & $12(15 \%)$ & $10(27 \%)$ \\
\hline \multicolumn{8}{|l|}{ Histology } \\
\hline non-squamous non-small & 107 (51\%) & $76(56 \%)$ & $29(40 \%)$ & $39(70 \%)$ & $14(42 \%)$ & $36(44 \%)$ & $16(43 \%)$ \\
\hline Squamous & $51(24 \%)$ & $26(19 \%)$ & $24(33 \%)$ & $9(16 \%)$ & $6(18 \%)$ & $20(25 \%)$ & $14(38 \%)$ \\
\hline SCLC & $53(25 \%)$ & $33(24 \%)$ & $20(27 \%)$ & $8(14 \%)$ & $13(39 \%)$ & $25(31 \%)$ & $7(19 \%)$ \\
\hline \multicolumn{8}{|l|}{ Staging } \\
\hline $3 A$ & $26(12 \%)$ & $19(14 \%)$ & $7(9.6 \%)$ & $7(12 \%)$ & $1(3.0 \%)$ & $13(16 \%)$ & $4(11 \%)$ \\
\hline 3B & 35 (17\%) & $21(16 \%)$ & $13(18 \%)$ & $11(20 \%)$ & 7 (21\%) & $11(14 \%)$ & $6(16 \%)$ \\
\hline $3 C$ & $13(6.2 \%)$ & $6(4.4 \%)$ & $7(9.6 \%)$ & $2(3.6 \%)$ & $3(9.1 \%)$ & $4(4.9 \%)$ & $4(11 \%)$ \\
\hline $4 \mathrm{~A}$ & $66(31 \%)$ & $45(33 \%)$ & $21(29 \%)$ & $17(30 \%)$ & $7(21 \%)$ & $29(36 \%)$ & $11(30 \%)$ \\
\hline 4B & $71(34 \%)$ & $44(33 \%)$ & $25(34 \%)$ & $19(34 \%)$ & $15(45 \%)$ & $24(30 \%)$ & $12(32 \%)$ \\
\hline $\begin{array}{l}\text { Targetable driver gene } \\
\text { mutation }^{\ddagger}\end{array}$ & $28(30 \%)$ & $23(34 \%)$ & $4(16 \%)$ & $18(50 \%)$ & $1(9.1 \%)$ & $6(20 \%)$ & $2(13 \%)$ \\
\hline (Missing) & 117 & 68 & 48 & 20 & 22 & 51 & 22 \\
\hline PD-L1 positive & $105(78 \%)$ & 64 (77\%) & 39 (81\%) & 30 (79\%) & 15 (79\%) & $36(75 \%)$ & $22(81 \%)$ \\
\hline (Missing) & 77 & 52 & 25 & 18 & 14 & 33 & 10 \\
\hline \multicolumn{8}{|l|}{ Treatment } \\
\hline chemoradiotherapy & $33(16 \%)$ & $19(14 \%)$ & 13 (18\%) & 10 (18\%) & $8(24 \%)$ & $10(12 \%)$ & $4(11 \%)$ \\
\hline chemotherapy & $126(60 \%)$ & $83(61 \%)$ & 41 (56\%) & 31 (55\%) & $17(52 \%)$ & $51(63 \%)$ & $24(65 \%)$ \\
\hline radiotherapy & $6(2.8 \%)$ & $3(2.2 \%)$ & $3(4.1 \%)$ & $1(1.8 \%)$ & $0(0 \%)$ & $3(3.7 \%)$ & $2(5.4 \%)$ \\
\hline
\end{tabular}

Median (IQR); Number (\%)

SCLC, Small Cell Lung Cancer; PD-L1, Programmed Death Ligand 1; BSC, Best Supportive Care.

* Three patients were excluded due to missingness

† Four patients were excluded due to missingness

‡ Including Epidermal Growth Factor Receptor (EGFR) gene mutation, Anaplastic Lymphoma Kinase (ALK) translocation, and c-ros oncogene 1 receptor tyrosine kinase (ROS1) translocation 


\begin{tabular}{|c|c|c|c|c|c|c|}
\hline \multirow[b]{2}{*}{$46(22 \%)$} & \multicolumn{2}{|c|}{ Social Isolation* } & \multicolumn{4}{|c|}{ Loneliness $^{\dagger}$} \\
\hline & $30(22 \%)$ & $16(22 \%)$ & $14(25 \%)$ & $8(24 \%)$ & $17(21 \%)$ & $7(19 \%)$ \\
\hline \multicolumn{7}{|l|}{ Median (IQR); Number (\%) } \\
\hline \multicolumn{7}{|c|}{ SCLC, Small Cell Lung Cancer; PD-L1, Programmed Death Ligand 1; BSC, Best Supportive Care. } \\
\hline \multicolumn{7}{|c|}{ * Three patients were excluded due to missingness } \\
\hline \multicolumn{7}{|c|}{ † Four patients were excluded due to missingness } \\
\hline $\begin{array}{l}\text { \# Including Epidermal Growth Factor Re } \\
\text { oncogene } 1 \text { receptor tyrosine kinase (RC }\end{array}$ & $\begin{array}{l}r(E G F R) c \\
\text { ranslocat }\end{array}$ & nutation, $A$ & lastic Lymp & na Kinas & inslocatic & -ros \\
\hline
\end{tabular}

Kaplan-Meier survival curves for all patients and stratified by social isolation and loneliness are shown in Figures 2, 3, and 4, respectively. These survival curves indicated that social isolation was associated with poorer OS. However, no difference in OS was observed between the groups. The results of the Cox proportional-hazards models for OS are summarized in Table 2. The hazard ratios of social isolation were 2.25 [ $95 \%$ confidence interval (Cl); 0.88 to 5.76 ] in the univariate analysis, $3.36(95 \% \mathrm{Cl} ; 1.36$ to 9.36$)$ in the multivariate analysis with complete case, and 3.47 (95\% Cl: 1.08 to 11.1 ] in the multivariate analysis with multiple imputation.

Table 2

Cox proportional hazard models for overall survival / death at home

\begin{tabular}{|c|c|c|c|}
\hline & $\begin{array}{l}\text { Univariate } \\
\text { analysis }\end{array}$ & $\begin{array}{l}\text { Multivariate analysis* with complete } \\
\text { case }\end{array}$ & Multivariate analysis* with multiple imputation \\
\hline \multicolumn{4}{|l|}{ Overall Survival } \\
\hline Social isolation & 1.65 (1.12 to 2.44$)$ & 1.87 (1.15 to 3.03$)$ & $1.40(0.916$ to 2.13$)$ \\
\hline \multicolumn{4}{|l|}{$\begin{array}{l}\text { Presence vs. } \\
\text { Absence }\end{array}$} \\
\hline \multicolumn{4}{|l|}{ Loneliness } \\
\hline q2 vs. q1 & 1.73 (0.93 to 3.22 ) & 1.90 (0.88 to 4.10$)$ & 1.23 (0.62 to 2.41$)$ \\
\hline q3 vs. q1 & 1.35 (0.81 to 2.25$)$ & 1.07 (0.52 to 2.19$)$ & 0.99 (0.55 to 1.78$)$ \\
\hline q4 vs. q1 & 1.58 (0.86 to 2.88$)$ & 1.20 (0.54 to 2.66$)$ & 1.15 (0.60 to 2.2$)$ \\
\hline \multicolumn{4}{|l|}{ Death at home } \\
\hline Social isolation & 2.25 (0.88 to 5.76$)$ & 3.36 (1.36 to 9.36$)$ & 3.47 (1.08 to 11.1$)$ \\
\hline \multicolumn{4}{|l|}{$\begin{array}{l}\text { Presence vs. } \\
\text { Absence }\end{array}$} \\
\hline \multicolumn{4}{|l|}{ Loneliness } \\
\hline q2 vs. q1 & 1.03 (0.23 to 4.56$)$ & 0.93 (0.20 to 4.38$)$ & 0.69 (0.08 to 5.65$)$ \\
\hline q3 vs. q1 & 1.01 (0.29 to 3.49 ) & 0.92 (0.25 to 3.41$)$ & 1.60 (0.56 to 4.54$)$ \\
\hline q4 vs. q1 & 1.2 (0.29 to 4.95$)$ & 1.26 (0.26 to 6.21$)$ & 1.06 (0.36 to 2.79$)$ \\
\hline \multicolumn{4}{|c|}{ Hazard ratio (95\%confidence intervals) } \\
\hline \multicolumn{4}{|c|}{$\begin{array}{l}\text { Social isolation was evaluated with Japanese version of the Lubben Social Network Scale (LSNS-6). Patients were divided by the cutoff of } \\
\text { English version. Loneliness was evaluated with Japanese Version } 3 \text { of the UCLA (University of California, Los Angeles) Loneliness Scale. } \\
\text { Higher score means worse loneliness. Patients were divided into quartiles. }\end{array}$} \\
\hline \multicolumn{4}{|c|}{$\begin{array}{l}\text { * adjusted variables: gender (male vs. female), age ( } \geq 71 \text { vs. } 70 \geq \text { ), smoke status (current or ex-smoker), performance status }(\geq 2 \text { vs. } 1 \geq \text { ), } \\
\text { TNM staging (stage } 4 \text { vs. stage3), histology (non-squamous non-small vs. squamous vs. small cell lung carcinoma), treatment } \\
\text { (chemoradiotherapy, chemotherapy, radiotherapy vs. best supportive care) }\end{array}$} \\
\hline
\end{tabular}

\section{Death at home}

A total of 102 patients died during the study period. The number of deaths at home were five (22\%), four (22\%), nine (22\%) and five (25\%) in the Ioneliness quartiles 1, 2, 3 and 4, respectively. Additionally, 10 (16\%) from the group without social isolation and 11 (28\%) from the group with social isolation died at home. The logistic regression models for death at home are summarized in Table 2 . In the multivariate analysis with multiple imputation, the odds ratios of death at home with social isolation were $2.25(95 \% \mathrm{Cl} ; 0.88 \cdot 5.76)$ in the univariate analysis, $3.36(95 \%$ 
$\mathrm{Cl} ; 1.36 \cdot 9.36)$ in the multivariate analysis with complete case and $3.47(95 \% \mathrm{Cl} ; 1.08 \cdot 11.1)$ in the multivariate analysis with multiple imputation. Loneliness was not associated with OS in univariate analysis, multivariate analysis with complete cases, and multiple imputation.

\section{Discussion Key results}

We investigated the relationship between loneliness and social isolation with OS and death at home in Japanese patients with advanced lung cancer. Our study suggested that social isolation could be related to shorter OS and more death at home, while loneliness was not related to OS and death at home.

\section{Interpretation}

To the best of our knowledge, this is the first study to suggest a relationship between social isolation and shorter OS in patients with lung cancer. Our previous study used the same dataset and showed no difference in treatment between patients with or without social isolation [13]. Therefore, it is speculated that the OS difference was not due to treatment choice, but owing to opportunities to access medical services or quality of home care. A systematic review showed that some determinants of hospital visits were regular sources of care and family resources [22]. This indicated that other people noticed a change in the patient's condition and encouraged hospital visits if the patient was socially connected. Evaluation of social isolation at the time of diagnosis might contribute to better prognosis.

There was no difference in OS and death rates in hospitals with or without loneliness. It is speculated that patients' feelings of loneliness did not change their medical access or home care. A systematic review showed that patients' mental condition related to hospital visits were attitudes toward health and not loneliness [22].

We found that social isolation was related to death at home. No previous study has investigated the relationship between social isolation and death at home in patients with advanced lung cancer. Obviously, caregivers are necessary for death at home [23]. In the case of a patient living alone whose relatives take care of the patient, they can wish to spend more time with the patient at home [24]. However, in the case of socially isolated patients, neighbors or adult guardians may recommend hospitalization. Further studies are required to assess the quality of death among socially isolated patients with advanced lung cancer.

\section{Generalizability}

Our inclusion criteria may have caused a selection bias. Some higher-income patients who preferred receiving more advanced treatments in specialized hospitals were not included. Patients who were clinically diagnosed with lung cancer, but did not visit the tertiary hospital for various reasons, were excluded. In such cases, the relationship between social factors and prognosis may become weaker.

In addition, this study was conducted in a country under a universal health insurance system and might be applicable to countries with the same system. Further cohort studies with other insurance systems are warranted.

\section{Strengths and limitations}

This study has two limitations. First, due to the slow recruitment rate of patients, we could not include enough patients as defined in the protocol. A small sample size may lead to beta errors. Hence, we could not estimate enough narrow confidence intervals. Second, social isolation and loneliness at the time of diagnosis may have changed during the observation period. However, social isolation or loneliness were speculated to be stable or worsened with time among patients with cancer. Further studies that evaluate the changes over time are necessary.

\section{Conclusion}

Our study suggests that social isolation may be related to OS and death at home among patients with advanced lung cancer, while loneliness does not seem to be related. Medical staff should pay attention to the social isolation of patients with advanced lung cancer at the time of their diagnosis.

\section{Declarations}

Funding: This research received no specific grant from any funding agency in the public, commercial, or not-for-profit sectors.

Conflicts of interest: The Authors declare that there is no conflict of interest.

Availability of data and material: The data supporting the results of this study are available from the corresponding author upon reasonable request. 


\section{Authors' contributions:}

(I) Conception and design: Tomoyasu Takemura, Yuki Kataoka, Toru Naganuma; (II) Administrative support: Kazuo Endo, Masataka Hirabayashi; (III) Provision of study materials or patients: All authors Except for Toru Naganuma; (IV) Collection and assembly of data: All Authors except for Toru Naganuma; (V) Data analysis and interpretation: Tomoyasu Takemura, Yuki Kataoka, Toru Naganuma; (VI) Manuscript writing: All authors; (VII) Final approval of manuscript: All authors

Ethics approval: The protocol was approved by the Hyogo Prefectural Amagasaki General Medical Center Institutional Review Board (\#29-164).

Consent to participate: Informed consent was obtained from all individual participants included in the study.

\section{Acknowledgement}

We are grateful to Kyoko Wasai for providing professional advice concerning the planning of this research and Editage (www.editage.jp) for English language editing.

\section{References}

1. The College of Family Physicians of Canada (Accessed August 30, 2021) Best Advice Social Determinants of Health, published March 2015. Available online: http://patientsmedicalhome.ca/files/uploads/BA_SocialD_ENG_WEB.pdf\#search=\%27ollege+of+family+physicians+of+canada+SDH\%27.

2. World Health Organization. Rio Political Declaration on Social Determinants of Health, published October 21 (2011) Available online: http://www.who.int/sdhconference/declaration/Rio_political_declaration.pdf?ua=1. (Accessed August 30, 2021)

3. Alcaraz Kl, Wiedt TL, Daniels EC et al (2020) Understanding and addressing social determinants to advance cancer health equity in the United States: A blueprint for practice, research, and policy. CA Cancer J Clin 70:31-46

4. Hurtado JL, Bacigalupe A, Calvo M et al (2015) Social inequalities in a population based colorectal cancer screening programme in the Basque Country. BMC Public Health 15:1021

5. Steven J, Kittner P, Sekar ME, Comeau et al (2021) Ethnic and Racial Variation in Intracerebral Hemorrhage Risk Factors and Risk Factor Burden. JAMA Netw Open 4(8):e2121921. doi: 10.1001/jamanetworkopen.2021.21921

6. Malcolm M, Frost H, Cowie J (2019) Loneliness and social isolation causal association with health-related lifestyle risk in older adults: a systematic review and meta-analysis protocol. Syst Rev 8:48

7. Holt-Lunstad J, Smith TB, Baker M et al (2015) Loneliness and social isolation as risk factors for mortality: a meta-analytic review [abstract]. Perspect Psychol Sci 10:227-237

8. Hyland KA, Small BJ, Gray JE et al (2019) Loneliness as a mediator of the relationship of social cognitive variables with depressive symptoms and quality of life in lung cancer patients beginning treatment [abstract]. Psychooncology 28:1234-1242

9. Finke I, Behrens G, Schwettmann L et al (2020) Socioeconomic differences and lung cancer survival in Germany: Investigation based on population-based clinical cancer registration [abstract]. Lung Cancer 142:1-8

10. Pezzi TA, Schwartz DL, Mohamed ASR et al (2018) Barriers to combined-modality therapy for limited-stage small-cell lung cancer. JAMA Oncol 4:e174504

11. Tendler S, Holmqvist M, Wagenius $G$ et al (2020) Educational level, management and outcomes in small-cell lung cancer (SCLC): A population-based cohort study [abstract]. Lung Cancer 139:111-117

12. Forrest LF, Adams J, Wareham H et al (2013) Socioeconomic inequalities in lung cancer treatment: systematic review and meta-analysis. PLoS Med 10:e1001376

13. Tomoyasu Takemura Y, Kataoka C, Shirakawa et al (2021 Jun) Influence of loneliness and social isolation on the diagnosis and treatment of Japanese patients with advanced lung cancer: a prospective cohort study. Ann Palliat Med 10(6):6236-6246. doi: 10.21037/apm-21402. Epub 2021 Jun 2

14. Takemura T, Yuki K, Koya O et al (2018) Influence of social determinants of health on patients with advanced lung cancer: a prospective cohort study. BMJ Open 8:e023152

15. Nanami Ashi Y, Kataoka T, Takemura et al (2020 Dec) Factors Influencing Social Isolation and Loneliness Among Lung Cancer Patients: A Cross-sectional Study. Anticancer Res 40(12):7141-7145. doi: 10.21873/anticanres.14744

16. Union for International Cancer Control. TMN classification of malignant tumours, eighth edition (2017) Available online: https://www.wiley.com/en-ad/TNM+Classification+of+Malignant+Tumours\%2C+8th+Edition-p-9781119263579. (Accessed August 30, 
2021)

17. Kurimoto A, Awata S, Ohkubo T et al (2011) Reliability and validity of the Japanese version of the abbreviated Lubben Social Network Scale. Nihon Ronen Igakkai Zasshi 48:149-157

18. Shioda A, Tadaka E, Okochi A (2017) Reliability and validity of the Japanese version of the Community Integration Measure for community-dwelling people with schizophrenia. Int J Ment Health Syst 11:29

19. EndLonelinessUK (Accessed August 30, 2021) Measuring your impact on loneliness in later life. Available online: https://www.campaigntoendloneliness.org/wp-content/uploads/Loneliness-Measurement-Guidance1.pdf.

20. Suzuki T (2009) Manual about life function assessment for care provision. Available online: http://www.mhlw.go.jp/topics/2009/05/dl/tp0501-1c_0001.pdf. (Accessed August 30, 2021)

21. Rubin DB (2004) Multiple imputation for nonresponse in surveys. John Wiley \& Sons, p 81

22. Jane McCusker I, Karp S, Cardin et al (2003) Determinants of emergency department visits by older adults: a systematic review. Determinants of emergency department visits by older adults: a systematic review. Acad Emerg Med 10(12):1362-1370. doi: 10.1111/j.1553-2712.2003.tb00011.x

23. Bruera AAlonso-BabarroE, Varela-Cerdeira et al (2011) Can this patient be discharged home? Factors associated with at-home death among patients with cancer. J Clin Oncol. Mar 20;29(9):1159-67. doi: 10.1200/JC0.2010.31.6752. Epub 2011 Feb 22

24. Kotaro Hashimoto K, Sato M, Kawahara et al (2018) Current State of Home Palliative Care and Factors Influencing Death at Home for Terminally III Cancer Patients Living in Single-person Households. Palliat Care Res 13(1):39-48

\section{Figures}

\section{Assessed for eligibility \\ from April 2018 to March $2020(n=269)$}

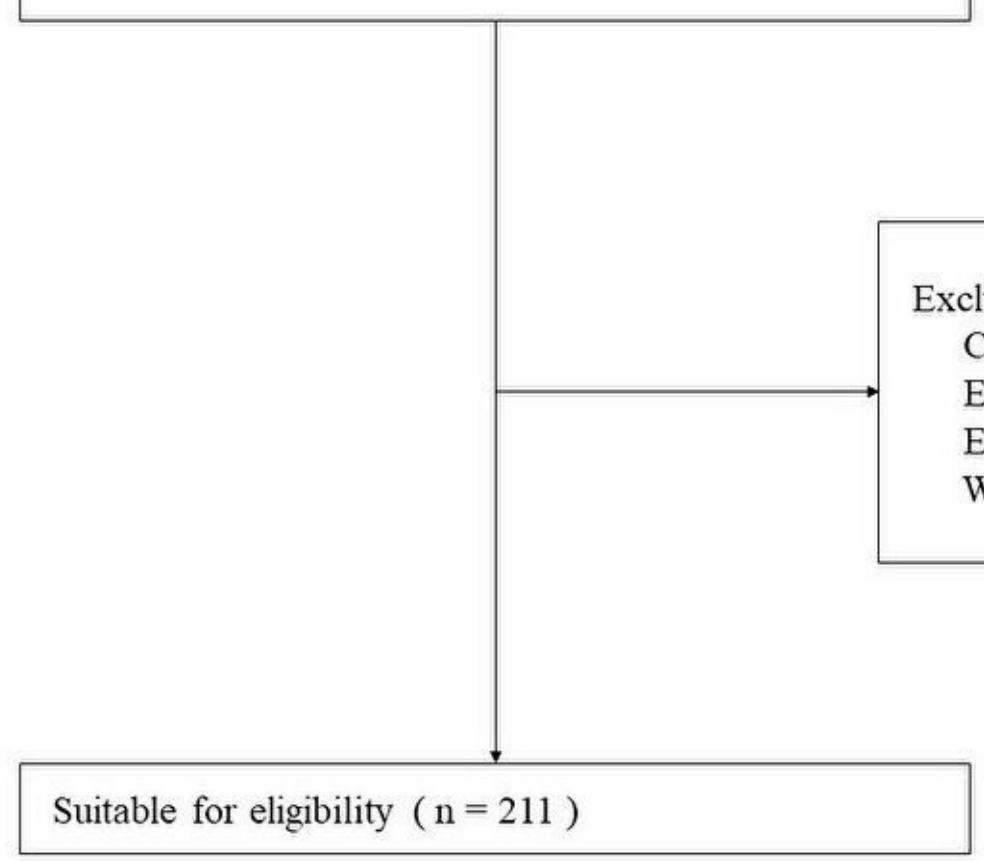

Figure 1 


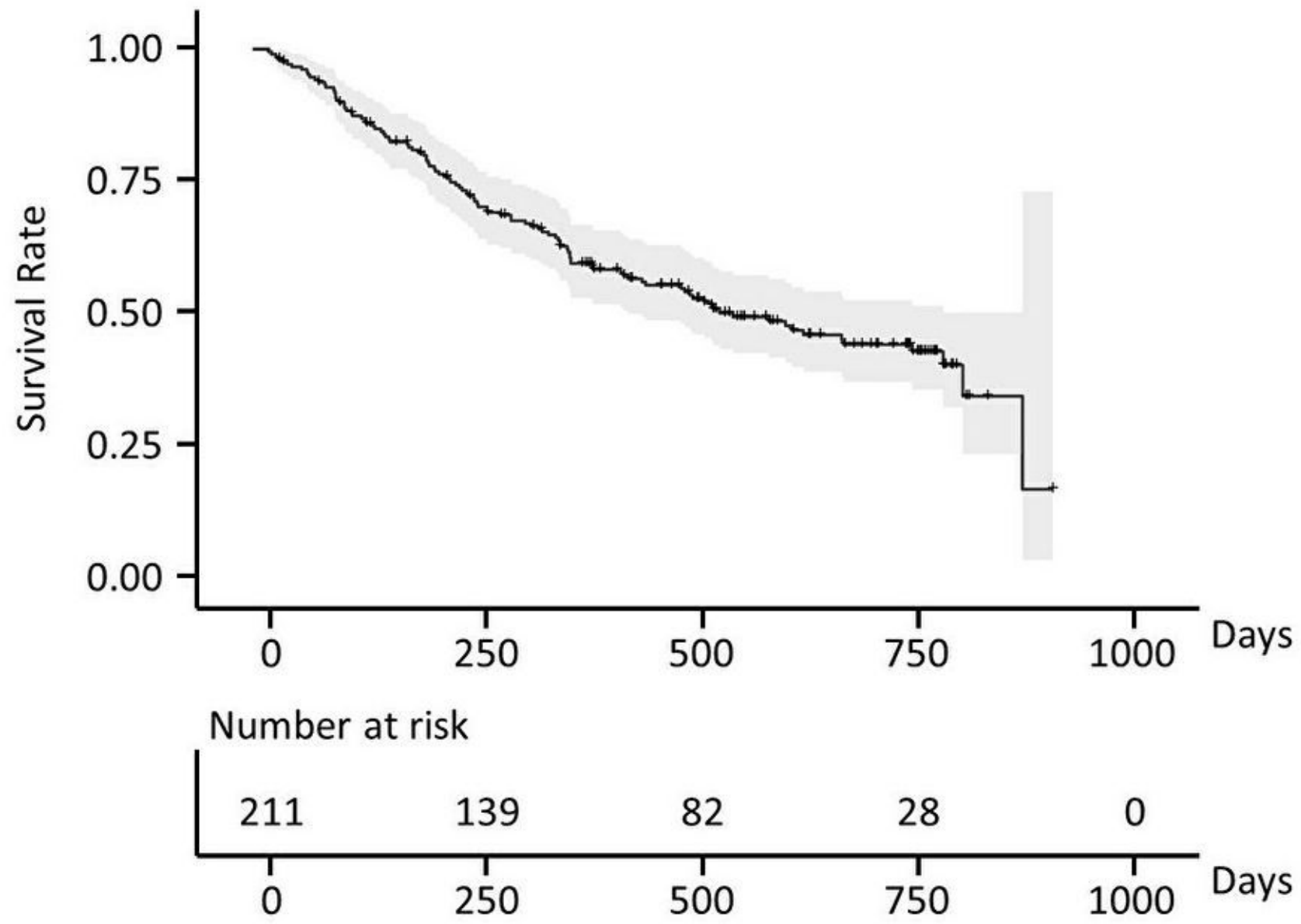

Figure 2

Overall survival of all patients 

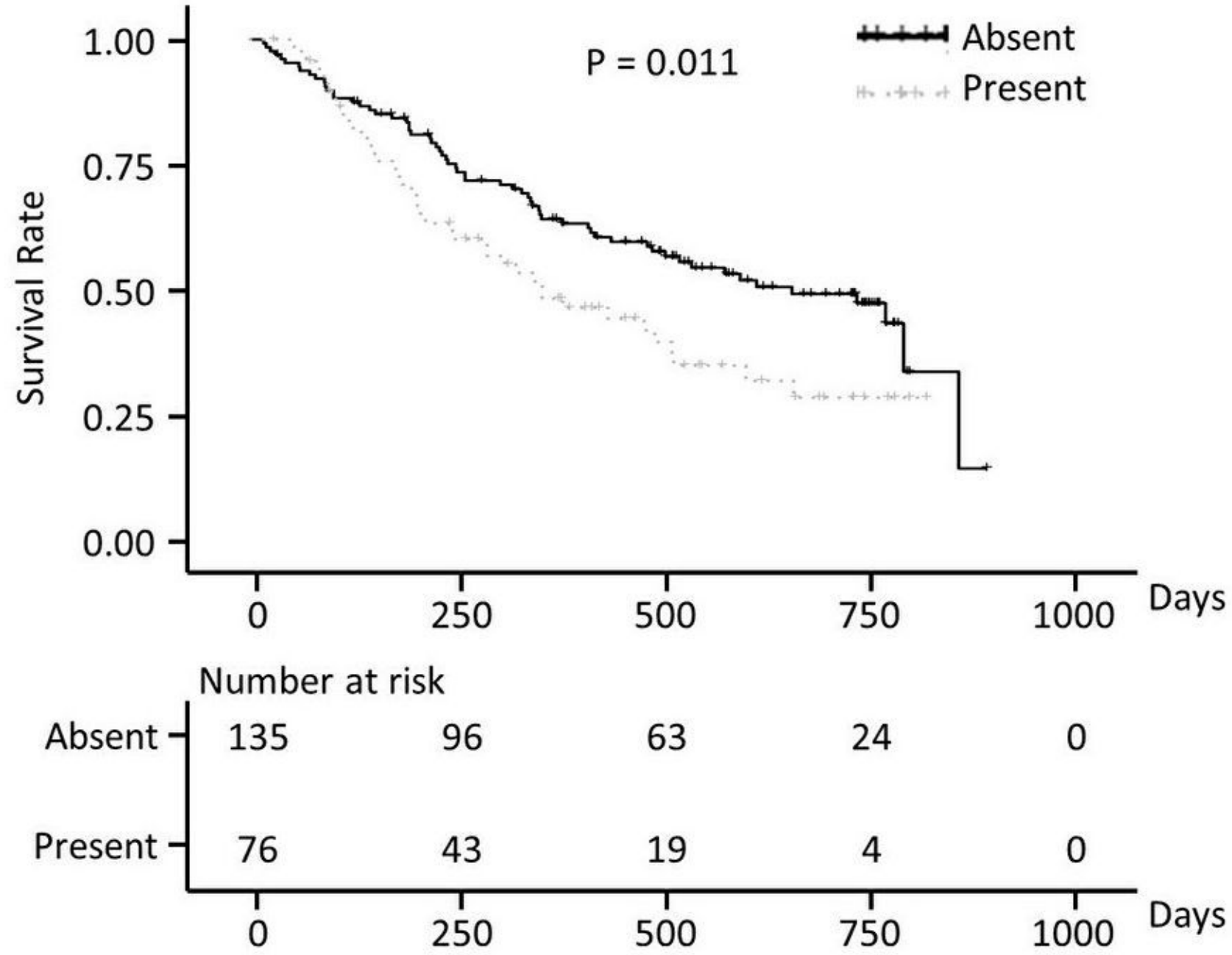

Figure 3

Kaplan-Meier survival curves for patients with lung cancer stratified by social isolation

p-value between with and without social isolation was evaluated by the log-rank test. 


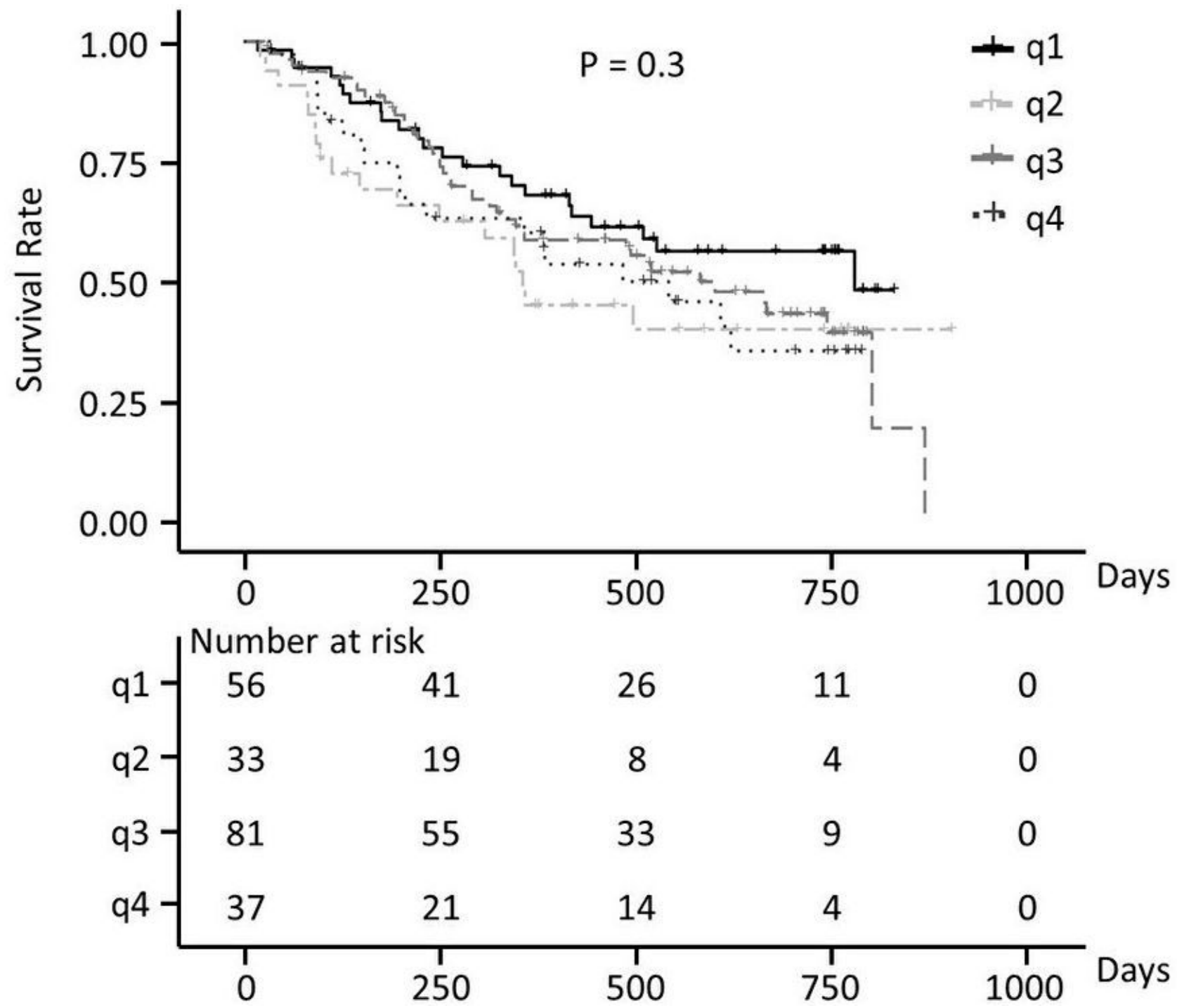

Figure 4

Kaplan-Meier survival curves for patients with lung cancer stratified by loneliness

p-value between with and without social isolation was evaluated by the log-rank test. 